Research Article

\title{
A New Single-Valued Neutrosophic Rough Sets and Related Topology
}

\author{
Qiu Jin, Kai Hu (D), Chunxin Bo, and Lingqiang Li $\mathbb{B}$ \\ School of Mathematical Sciences, Liaocheng University, Liaocheng 252059, China \\ Correspondence should be addressed to Kai Hu; hukai80@126.com and Lingqiang Li; lilingqiang@lcu.edu.cn
}

Received 1 March 2021; Revised 19 June 2021; Accepted 5 July 2021; Published 17 July 2021

Academic Editor: Yongqiang Fu

Copyright (C) 2021 Qiu Jin et al. This is an open access article distributed under the Creative Commons Attribution License, which permits unrestricted use, distribution, and reproduction in any medium, provided the original work is properly cited.

\begin{abstract}
(Fuzzy) rough sets are closely related to (fuzzy) topologies. Neutrosophic rough sets and neutrosophic topologies are extensions of (fuzzy) rough sets and (fuzzy) topologies, respectively. In this paper, a new type of neutrosophic rough sets is presented, and the basic properties and the relationships to neutrosophic topology are discussed. The main results include the following: (1) For a single-valued neutrosophic approximation space $(U, R)$, a pair of approximation operators called the upper and lower ordinary single-valued neutrosophic approximation operators are defined and their properties are discussed. Then the further properties of the proposed approximation operators corresponding to reflexive (transitive) single-valued neutrosophic approximation space are explored. (2) It is verified that the single-valued neutrosophic approximation spaces and the ordinary single-valued neutrosophic topological spaces can be interrelated to each other through our defined lower approximation operator. Particularly, there is a one-to-one correspondence between reflexive, transitive single-valued neutrosophic approximation spaces and quasidiscrete ordinary single-valued neutrosophic topological spaces.
\end{abstract}

\section{Introduction}

The original notion of neutrosophic set was proposed by Smarandache [1]. For the convenience of application, Wang et al. [2] investigated the single-valued neutrosophic set (Svns). In Svns, three independent membership functions (truth, indeterminacy, and falsity) are considered; hence, it can be regarded as extensions of fuzzy set [3] and intuitionistic fuzzy set [4]. There are many works on the theory and application of Svns (see Abdel-Basset [5], Ye [6, 7], Samant [8], Yang [9, 10], Zhang [11-13], Zavadskas [14], and $\mathrm{Xu}$ [15] as well as Peng's review paper [16]).

The fusion of neutrosophic sets with rough sets theory [17] is an important research direction. According to Li's review paper [18], there exists two fundamental combinations of rough sets and neutrosophic sets: Broumi's rough neutrosophic sets [19] and Sweety's neutrosophic rough sets [20]. Many other models can be regarded as their extensions $[12,21-24]$. (i) Broumi's rough neutrosophic sets [19]: let $R$ be an equivalent relation (can be easily extended for an arbitrary binary relation) on $U$. Then, for each neutrosophic set $A$ on $U$, a pair of neutrosophic sets $\underline{R}(A)$ and $\bar{R}(A)$ on $U$ are defined as the lower and upper approximations of $A$ w.r.t. $(U, R)$.

(ii) Sweety's neutrosophic rough sets [20]: let $R$ be a neutrosophic relation on $U$. Then, for each neutrosophic set $A$ on $U$, a pair of neutrosophic sets $\underline{R}(A)$ and $\bar{R}(A)$ on $U$ are defined as the lower and upper approximations of $A$ w.r.t. $(U, R)$. Yang [10] defined a similar model by considering the singlevalued neutrosophic relation and single-valued neutrosophic set on $U$.

In this paper, we shall introduce a new model of rough sets fusion with neutrosophic sets under the framework of single-valued neutrosophic approximation space $(U, R)$ (i.e., a nonempty set $U$ together with a single-valued neutrosophic relation $R$ on $U$ ). For each ordinary subset $A$ of $U$, we shall 
define a pair of single-valued neutrosophic sets $\underline{R}(A)$ and $\bar{R}(A)$ on $U$ as the lower and upper approximations of $A$ with respect to $(U, R)$. Obviously, our model is different from Broumi-Sweety-Yang's models, since, in our model, the original sets are ordinary subsets of $U$ and their approximations are single-valued neutrosophic sets, but, in Broumi-Sweety-Yang's models, the original sets and their approximations are all (single-valued) neutrosophic sets. Hence, our rough sets will be called ordinary single-valued neutrosophic rough sets.

(Fuzzy) rough sets are closely related to (fuzzy) topology [25-42]. The well-known result may be that there is a one-toone correspondence between reflexive and transitive (fuzzy) approximation spaces and quasidiscrete (fuzzy) topological spaces $[26,37,38]$. Under the framework of single-valued neutrosophic sets, two kinds of neutrosophic topological spaces are discussed (for more general neutrosophic topology, refer to Al-Omeri [43] and Lupianez [44]).

(i) Yang's single-valued neutrosophic topological spaces [45]: for a nonempty set $U$, Yang defined the single-valued neutrosophic topology on $U$ as a subset $\tau$ of Svns $(U)$ (the set of all single-valued neutrosophic sets on $U$ ) with some conditions. Yang's space can be regarded as an extension of Lowen's fuzzy topological space [46]. Yang also proved that there is a one-to-one correspondence between reflexive and transitive single-valued neutrosophic approximation spaces and his single-valued neutrosophic rough topological spaces.

(ii) Kim's ordinary single-valued neutrosophic topological spaces [47]: for a nonempty set $U$, Kim defined the ordinary single-valued neutrosophic topology on $U$ as a neutrosophic set $\tau$ on $P(U)$ (the power set of $U$ ) with some conditions. Kim's space can be regarded as an extension of Sostak's fuzzy topology [48] (or Ying's fuzzifying topology [49]).

In this paper, we shall prove that there are close relationships between our ordinary single-valued neutrosophic rough sets and Kim's ordinary single-valued neutrosophic topological spaces. The close relationships exhibit that it is meaningful to investigate the new rough sets model.

The method of this paper and the comparison with related literature can be summarized in Table 1 .

The remainder of this paper is organized as follows. In Section 2, we will recall some knowledge about neutrosophic sets and rough sets. In Section 3, we shall give the notion of ordinary single-valued neutrosophic upper and lower approximation operators and discuss their properties. Then we will explore the further properties of the proposed approximations corresponding to reflexive (transitive) single-valued neutrosophic approximation space. In Section 4, we will prove that each single-valued neutrosophic approximation space induces an ordinary single valued neutrosophic topological space via our defined lower approximation. In Section 5, we shall verify that each ordinary single-valued neutrosophic topological space induces a single-valued neutrosophic approximation space. In Section 6, we will show that there is a one-to-one correspondence between reflexive and transitive singlevalued neutrosophic approximation spaces and quasidiscrete ordinary single-valued neutrosophic topological spaces.

\section{Preliminaries}

In this section, we recall some knowledge about neutrosophic rough sets and neutrosophic topologies used in this paper.

Unless otherwise stated, we always assume that $U$ is a nonempty infinite set. We denote $P(U)$ as the power set of $U$ and define $A^{c}=U-A$ for $A \in P(U)$.

Definition 1 (see [2]). An Svns $A=\left(A_{T}, A_{I}, A_{F}\right)$ on $U$ is defined as three membership functions $A_{T}, A_{I}, A_{F}: U \longrightarrow[0,1]$, which are interpreted as truthmembership function, indeterminacy-membership function, and falsity-membership function, respectively. All Svnss are denoted by $\operatorname{Svns}(U)$.

Each $\alpha=\left(\alpha_{1}, \alpha_{2}, \alpha_{3}\right) \in[0,1]^{3}$ is called a single-valued neutrosophic number, and its complement is defined as $\alpha^{c}=\left(\alpha_{3}, 1-\alpha_{2}, \alpha_{1}\right)$. We denote the single-valued neutrosophic numbers $T=(1,0,0)$ and $\perp=(0,1,1)$. Obviously, $T^{c}=\perp$ and $\perp^{c}=T$.

Remark 1. Pythagorean fuzzy set [50] is also an important extension of intuitionistic fuzzy set. We can observe that when restricting $0 \leq\left(A_{T}(x)\right)^{2}+\left(A_{F}(x)\right)^{2} \leq 1$ and $A_{I}(x)=\sqrt{1-\left(A_{T}(x)\right)^{2}-\left(A_{F}(x)\right)^{2}}$, an Svns becomes a Pythagorean fuzzy set.

For $A \in P(U)$, we define $\mathrm{T}_{A} \in \operatorname{Svns}(U)$ as follows: $\forall x \in U, \top_{A}(x)=\top$ if $x \in A$ and $\mathrm{\top}_{A}(x)=\perp$ if $x \notin A$.

Definition 2 (see $[2,6,10])$. Let $A, B, A_{j}(j \in J) \in \operatorname{Svns}(U)$.

(1) We denote $A \sqsubseteq B$ if, for any $x \in U, A_{T}(x) \leq B_{T}(x)$, $A_{I}(x) \geq B_{I}(x)$, and $A_{F}(x) \geq B_{F}(x)$. By $A=B$, we mean $A \sqsubseteq B$ and $B \sqsubseteq A$

(2) We define $A^{c} \in \operatorname{Svns}(U)$ as $\forall x \in U, A^{c}$ $(x)=(A(x))^{c}=\left(A_{F}(x), 1-A_{I}(x), A_{T}(x)\right)$

(3) We define $\sqcup_{j \in J} A_{j}, \Pi_{j \in J} A_{j} \in \operatorname{Svns}(U)$ by $\forall x \in U$,

$$
\begin{aligned}
& \left(\sqcup_{j \in J} A_{j}\right)(x)=\left(\vee_{j \in J}\left(A_{j}\right)_{T}(x), \wedge_{j \in J}\left(A_{j}\right)_{I}(x), \wedge_{j \in J}\left(A_{j}\right)_{F}(x)\right), \\
& \left(\sqcap_{j \in J} A_{j}\right)(x)=\left(\wedge_{j \in J}\left(A_{j}\right)_{T}(x), \vee_{j \in J}\left(A_{j}\right)_{I}(x), \vee_{j \in J}\left(A_{j}\right)_{F}(x)\right) .
\end{aligned}
$$

Definition 3 (see [10]). An Svns $R$ on $U \times U$ is referred to a single-valued neutrosophic relation (Svnr) on $U$. Then the pair $(U, R)$ is said to be a single-valued neutrosophic approximation space (Svnas). Furthermore, $R$ is called 
TABLE 1: Method and comparison.

\begin{tabular}{llcccc}
\hline Rough set & $R$ & $A$ & $\bar{R}(A), \underline{R}(A)$ & Topology $\tau_{R}$ \\
\hline Rough neutrosophic sets [19] & - & + & + & Yang's topology [45] & Yang's topology [45] \\
Neutrosophic rough sets [10, 20] & + & + & + & Kim's topology [47] \\
Our neutrosophic rough sets & + & - & + & $\sqrt{ }$ \\
\hline
\end{tabular}

Notes: "+" represents that the set is a single-valued neutrosophic set, and "-" represents that it is not; " $\sqrt{ }$ " represents that there is a bijection between the considered rough sets and topologies, and " $x$ " represents that there is no bijection.

(i) Reflexive if $\forall x \in U, \quad R(x, x)=\mathrm{T}, \quad$ i.e., $R_{T}(x, x)=1, R_{I}(x, x)=0, R_{F}(x, x)=0$ (ii) Transitive if $R_{T}, R_{I}^{c}, R_{F}^{c}$ are all transitive fuzzy relations, that is, $\forall x, y, z \in U$

$$
R_{T}(x, y) \wedge R_{T}(y, z) \leq R_{T}(x, z), R_{I}(x, y) \vee R_{I}(y, z) \geq R_{I}(x, z), R_{F}(x, y) \vee R_{F}(y, z) \geq R_{F}(x, z) .
$$

Definition 4 (see Definition 3.1 in $[10]$ ). Let $(U, R)$ be an Svnas. For $A \in \operatorname{Svns}(U)$, the upper and lower approximations of $A$, denoted by $\overline{Y R}(A), \underline{Y R}(A) \in \operatorname{Svns}(U)$, are defined as follows: $\forall x \in U$,

$$
\begin{aligned}
\overline{Y R}(A)_{T}(x) & =\bigvee_{y \in U}\left(R_{T}(x, y) \wedge A_{T}(y)\right), \\
\overline{Y R}(A)_{I}(x) & =\bigwedge_{y \in U}\left(R_{I}(x, y) \vee A_{I}(y)\right), \\
\overline{Y R}(A)_{F}(x) & =\bigwedge_{y \in U}\left(R_{F}(x, y) \vee A_{F}(y)\right), \\
\underline{Y R}(A)_{T}(x) & =\bigwedge_{y \in U}\left(R_{F}(x, y) \vee A_{T}(y)\right), \\
\underline{Y R}(A)_{I}(x) & =\bigvee_{y \in U}\left(\left(1-R_{I}(x, y)\right) \wedge A_{I}(y)\right), \\
\underline{Y R}(A)_{F}(x) & =\bigvee_{y \in U}\left(R_{T}(x, y) \wedge A_{F}(y)\right),
\end{aligned}
$$

The pair $(\overline{Y R}(A), \underline{Y R}(A))$ is referred to the singlevalued neutrosophic rough sets of $A . \overline{Y R}$ and $\underline{Y R}$ are said to be the single-valued neutrosophic upper and lower approximation operators, respectively.

Definition 5 (see Definition 8 in [47]). An Svns $\tau$ on $P(U)$, that is, $\tau=\left(\tau_{T}, \tau_{I}, \tau_{F}\right)$ with $\tau_{T}, \tau_{I}, \tau_{F}: P(U) \longrightarrow[0,1]$, is referred to an ordinary single-valued neutrosophic topology (OSvnt) on $U$ if $\tau$ fulfills the following conditions:

$($ OSvnt 1$) \tau(\varnothing)=\tau(U)=\mathrm{\top}$,

(OSvnt2)For any $A, B \in P(U)$,

$\tau_{T}(A \cap B) \geq \tau_{T}(A) \wedge \tau_{T}(B)$,

$\tau_{I}(A \cap B) \leq \tau_{I}(A) \vee \tau_{I}(B)$,

$\tau_{F}(A \cap B) \leq \tau_{F}(A) \vee \tau_{F}(B)$,

(OSvnt3)For any $A_{j}(j \in J) \in P(U)$,

$$
\begin{aligned}
& \tau_{T}\left(\cup_{j \in J} A_{j}\right) \geq \bigwedge_{j \in J} \tau_{T}\left(A_{j}\right), \\
& \tau_{I}\left(\cup_{j \in J} A_{j}\right) \leq \bigvee_{j \in J} \tau_{I}\left(A_{j}\right), \\
& \tau_{F}\left(\cup_{j \in J} A_{j}\right) \leq \bigvee_{j \in J} \tau_{F}\left(A_{j}\right) .
\end{aligned}
$$

The pair $(U, \tau)$ is said to be an ordinary single-valued neutrosophic topological space (OSvnts).

For examples and more results about OSvnts, refer to [47].

The following lemma can be easily observed. We will use it without mentioning again.

Lemma 1. Let $\alpha, \beta \in[0,1]$. Then the following conditions are equivalent:

(1) $\alpha \leq \beta$

(2) For all $\gamma \in[0,1), \gamma<\alpha \Rightarrow \gamma<\beta$

(3) For all $\gamma \in[0,1), \gamma<\alpha \Rightarrow \gamma \leq \beta$

(4) For all $\gamma \in(0,1], \gamma \leq \alpha \Rightarrow \gamma \leq \beta$

(5) For all $\gamma \in(0,1], \gamma>\beta \Rightarrow \gamma \geq \alpha$

\section{Ordinary Single-Valued Neutrosophic Rough Sets for Svnas}

In this section, we present the notions and properties of ordinary single-valued neutrosophic upper and lower approximation operators.

Definition 6. Let $(U, R)$ be an Svnas. For $A \in P(U)$, the upper and lower approximations of $A$, denoted by $\bar{R}(A), \underline{R}(A) \in \operatorname{Svns}(U)$, are defined as follows: $\forall x \in U$,

$$
\begin{aligned}
& \bar{R}(A)_{T}(x)=\bigvee_{y \in A} R_{T}(x, y), \\
& \bar{R}(A)_{I}(x)=\bigwedge_{y \in A} R_{I}(x, y), \\
& \bar{R}(A)_{F}(x)=\bigwedge_{y \in A} R_{F}(x, y), \\
& \underline{R}(A)_{T}(x)=\bigwedge_{y \notin A} R_{F}(x, y), \\
& \underline{R}(A)_{I}(x)=\bigvee_{y \notin A}\left(1-R_{I}(x, y)\right), \\
& \underline{R}(A)_{F}(x)=\bigvee_{y \notin A} R_{T}(x, y) .
\end{aligned}
$$


The pair $(\bar{R}(A), \underline{R}(A))$ is referred to the ordinary singlevalued neutrosophic rough sets of $A . \bar{R}$ and $\underline{R}$ are said to be the ordinary single-valued neutrosophic upper and lower approximation operators, respectively.

\section{Remark 2}

(1) The definition of $\bar{R}(A)_{T}(x)$ is an interpretation of the fact that "the join of $R_{T}(x)$ and $A$ is not empty," and the definition of $\underline{R}(A)_{T}(x)$ is an interpretation of the fact that " $R_{T}(x)$ is contained in $A$ (or equivalent, $A^{c}$ is contained in $R_{F}(x)$ )."

(2) For a fuzzy relation $r$ on $U$, it is easily observed that $r$ induces an Svnr $R_{r}$ on $U$ defined as follows: $\forall(x, y) \in U \times U,\left(R_{r}\right)_{T}(x, y)=r(x, y),\left(R_{r}\right)_{I}(x, y)$ $=0,\left(R_{r}\right)_{F}(x, y)=1-r(x, y)$. For $A \in P(U)$, we have $\overline{R_{r}}(A)_{T}=\mathrm{\vee}_{y \in A} r(x, y)=\bar{r}(A), R_{r}(A)_{T}=\vee_{y \notin A}$ $(1-r(x, y))=\underline{r}(A)$, where $\bar{r}(A), \underline{r}(\bar{A})$ are the fuzzy approximations of ordinary subset w.r.t. fuzzy relation in the work of Yao [51]. Therefore, the singlevalued neutrosophic approximations in this paper are a generalization of Yao's fuzzy approximations.

(3) Obviously, the single-valued neutrosophic approximation operators in this paper are different from the single-valued neutrosophic approximation operators in the work of Yang [10], since our operators are defined from $P(U)$ to Svns $(U)$ and Yang's operators are defined from $\operatorname{Svns}(U)$ to $\operatorname{Svns}(U)$.

Example 1. Let $(U, R)$ be an Svnas with $U=\left\{x_{1}, x_{2}, x_{3}\right\}$ and let $R$ be defined as in Table 2 .

Taking $A=\left\{x_{1}, x_{2}\right\}$, we have

$$
\begin{aligned}
& \underline{R}(A)_{T}\left(x_{1}\right)=R_{F}\left(x_{1}, x_{3}\right)=0.4, \\
& \underline{R}(A)_{I}\left(x_{1}\right)=1-R_{I}\left(x_{1}, x_{3}\right)=1, \\
& \underline{R}(A)_{F}\left(x_{1}\right)=R_{T}\left(x_{1}, x_{3}\right)=1, \\
& \underline{R}(A)_{T}\left(x_{2}\right)=R_{F}\left(x_{2}, x_{3}\right)=1, \\
& \underline{R}(A)_{I}\left(x_{2}\right)=1-R_{I}\left(x_{2}, x_{3}\right)=1, \\
& \underline{R}(A)_{F}\left(x_{2}\right)=R_{T}\left(x_{2}, x_{3}\right)=0.6, \\
& \underline{R}(A)_{T}\left(x_{3}\right)=R_{F}\left(x_{3}, x_{3}\right)=0, \\
& \underline{R}(A)_{I}\left(x_{3}\right)=1-R_{I}\left(x_{3}, x_{3}\right)=1, \\
& \underline{R}(A)_{F}\left(x_{1}\right)=R_{T}\left(x_{3}, x_{3}\right)=1,
\end{aligned}
$$

$$
\begin{aligned}
& \bar{R}(A)_{T}\left(x_{1}\right)=R_{T}\left(x_{1}, x_{1}\right) \vee R_{T}\left(x_{1}, x_{2}\right)=0 \vee 0.3=0.3, \\
& \bar{R}(A)_{I}\left(x_{1}\right)=R_{I}\left(x_{1}, x_{1}\right) \wedge R_{I}\left(x_{1}, x_{2}\right)=0 \wedge 0.1=0, \\
& \bar{R}(A)_{F}\left(x_{1}\right)=R_{F}\left(x_{1}, x_{1}\right) \wedge R_{F}\left(x_{1}, x_{2}\right)=1 \wedge 0.6=0.6, \\
& \bar{R}(A)_{T}\left(x_{2}\right)=R_{T}\left(x_{2}, x_{1}\right) \vee R_{T}\left(x_{2}, x_{2}\right)=0 \vee 0.6=0.6, \\
& \bar{R}(A)_{I}\left(x_{2}\right)=R_{I}\left(x_{2}, x_{1}\right) \wedge R_{I}\left(x_{2}, x_{2}\right)=0.2 \wedge 0.5=0.2, \\
& \bar{R}(A)_{F}\left(x_{2}\right)=R_{F}\left(x_{2}, x_{1}\right) \wedge R_{F}\left(x_{2}, x_{2}\right)=0.4 \wedge 1=0.4, \\
& \bar{R}(A)_{T}\left(x_{3}\right)=R_{T}\left(x_{3}, x_{1}\right) \vee R_{T}\left(x_{3}, x_{2}\right)=1 \vee 1=1, \\
& \bar{R}(A)_{I}\left(x_{3}\right)=R_{I}\left(x_{3}, x_{1}\right) \wedge R_{I}\left(x_{3}, x_{2}\right)=0 \wedge 0.5=0, \\
& \bar{R}(A)_{F}\left(x_{3}\right)=R_{F}\left(x_{3}, x_{1}\right) \wedge R_{F}\left(x_{3}, x_{2}\right)=1 \wedge 1=1 .
\end{aligned}
$$

Hence, we obtain $\underline{R}(A)$ and $\bar{R}(A)$ as in Table 3.

Theorem 1. Let $(U, R)$ be an Svnas. Then we have the following:

(1) $\underline{R}(U)=\mathrm{\top}_{U} ; \bar{R}(\varnothing)=\mathrm{\top}_{\varnothing}$

(2) If $A \subseteq B$, then $\underline{R}(A) \subseteq \underline{R}(B)$ and $\bar{R}(A) \sqsubseteq \bar{R}(B)$

(3) For all $A_{j}(j \in J) \in P(U), \underline{R}\left(\cap_{j \in J} A_{j}\right)=\Pi_{j \in J} \underline{R}\left(A_{j}\right)$ and $\bar{R}\left(\cup_{j \in J} A_{j}\right)=\cup_{j \in J} \bar{R}\left(A_{j}\right)$

(4) For $\quad A \in P(U), \quad \bar{R}(A)=\left(\underline{R}\left(A^{c}\right)\right)^{c} \quad$ and $\underline{R}(A)=\left(\bar{R}\left(A^{c}\right)\right)^{c}$

Proof. For (1)-(3), we prove only the results for lower approximation. The proofs for upper approximation are similar and hence are omitted.

(1) For any $x \in U$, we have $\underline{R}(U)_{T}(x)=\wedge_{y \notin U} R_{F}$ $(x, y)=1, \underline{R}(U)_{I}(x)=\vee_{y \notin U}\left(1-R_{I}(x, y)\right)=0, \underline{R}$ $(U)_{F}(x)=\vee_{y \notin U} R_{T}(x, y)=0$. Hence, $\underline{R}(U)=\mathrm{T}_{U}$.

(2) For any $x \in U$ and $A \subseteq B$, we obtain

$$
\begin{aligned}
& \underline{R}(A)_{T}(x)=\bigwedge_{y \notin A} R_{F}(x, y) \leq \bigwedge_{y \notin B} R_{F}(x, y)=\underline{R}(B)_{T}(x), \\
& \underline{R}(A)_{I}(x)=\bigvee_{y \notin A}\left(1-R_{I}(x, y)\right) \geq \bigvee_{y \notin B}\left(1-R_{I}(x, y)\right)=\underline{R}(B)_{I}(x), \\
& \underline{R}(A)_{F}(x)=\bigvee_{y \notin A} R_{T}(x, y) \geq \bigvee_{y \notin B} R_{T}(x, y)=\underline{R}(B)_{F}(x) .
\end{aligned}
$$


Hence, $\underline{R}(A) \underline{\underline{R}} \underline{R}(B)$.

(3) For any $x \in U$,

$$
\begin{aligned}
\left(\prod_{j \in J} \underline{R}\left(A_{j}\right)\right)_{T}(x) & =\bigwedge_{j \in J} \underline{R}\left(A_{j}\right)_{T}(x) \\
& =\bigwedge_{j \in J} \bigwedge_{y \notin A_{j}} R_{F}(x, y)=\bigwedge_{y \notin \cap_{j \in J} A_{j}} R_{F}(x)=\underline{R}\left(\cap_{j \in J} A_{j}\right)_{T}(x), \\
\left(\prod_{j \in J} \underline{R}\left(A_{j}\right)\right)_{I}(x) & =\bigvee_{j \in J} \underline{R}\left(A_{j}\right)_{I}(x) \\
& =\bigvee_{j \in J} \bigvee_{y \notin A_{j}}\left(1-R_{I}(x, y)\right)=\bigvee_{y \notin \cap_{j \in J} A_{j}}\left(1-R_{I}(x, y)\right)=\underline{R}\left(\cap_{j \in J} A_{j}\right)_{I}(x), \\
\left(\prod_{j \in J} \underline{R}\left(A_{j}\right)\right)_{F}(x) & =\bigvee_{j \in J} \underline{R}\left(A_{j}\right)_{F}(x) \\
& =\bigvee_{j \in J} \bigvee_{y \notin A_{j}} R_{T}(x, y)=\bigvee_{y \notin \cap_{j \in J} A_{j}} R_{T}(x, y)=\underline{R}\left(\cap_{j \in J} A_{j}\right)_{F}(x) .
\end{aligned}
$$

Hence, $\underline{R}\left(\cap_{j \in J} A_{j}\right)=\Pi_{j \in J} \underline{R}\left(A_{j}\right)$.

(4) For any $x \in U$,

$$
\begin{aligned}
& \left(\underline{R}\left(A^{c}\right)\right)_{T}^{c}(x)=\left(\underline{R}\left(A^{c}\right)\right)_{F}(x)=\bigvee_{y \in A} R_{T}(x, y)=\bar{R}(A)_{T}(x), \\
& \left(\underline{R}\left(A^{c}\right)\right)_{I}^{c}(x)=1-\left(\underline{R}\left(A^{c}\right)\right)_{I}(x)=1-\bigvee_{y \in A}\left(1-R_{I}(x, y)\right)=\bigwedge_{y \in A} R_{I}(x, y)=\bar{R}(A)_{I}(x), \\
& \left(\underline{R}\left(A^{c}\right)\right)_{F}^{c}(x)=\left(\underline{R}\left(A^{c}\right)\right)_{T}(x)=\bigwedge_{y \in A} R_{F}(x, y)=\bar{R}(A)_{F}(x) .
\end{aligned}
$$

Hence, $\bar{R}(A)=\left(\underline{R}\left(A^{c}\right)\right)^{c}$. That is, $\underline{R}(A)=\left(\bar{R}\left(A^{c}\right)\right)^{c}$ can be proved similarly.

The following theorem gives a characterization on the approximation operators generated by reflexive Svnas.

Theorem 2. Let $(U, R)$ be an Svnas. Then the following three are equivalent:

(1) $R$ is reflexive

(2) $\underline{R}(A) \underline{\complement} \top_{A}$ for each $A \in P(U)$
(3) $\mathrm{T}_{A} \sqsubseteq \bar{R}(A)$ for each $A \in P(U)$

Proof. (1) $\Rightarrow$ (2). If $x \in A$, then

$$
\begin{aligned}
\underline{R}_{T}(A)(x) \leq\left(\top_{A}\right)_{T}(x) & =1, \\
\underline{R}_{I}(A)(x) \geq\left(\top_{A}\right)_{I}(x) & =0, \\
\underline{R}_{F}(A)(x) \geq\left(\top_{A}\right)_{F}(x) & =0 .
\end{aligned}
$$

If $x \notin A$, then

$$
\begin{aligned}
& \underline{R}_{T}(A)(x)=\bigwedge_{y \notin A} R_{F}(x, y) \leq R_{F}(x, x) \stackrel{(1)}{=} 0=\left(\top_{A}\right)_{T}(x), \\
& \underline{R}_{I}(A)(x)=\bigvee_{y \notin A}\left(1-R_{I}(x, y)\right) \geq 1-R_{I}(x, x) \stackrel{(1)}{=} 1-0=1=\left(\top_{A}\right)_{I}(x), \\
& \underline{R}_{F}(A)(x)=\bigvee_{y \notin A}\left(R_{T}(x, y)\right) \geq R_{T}(x, x) \stackrel{(1)}{=} 1=\left(\top_{A}\right)_{F}(x) .
\end{aligned}
$$

Hence, $\underline{R}(A) \sqsubseteq \mathrm{T}_{A}$.

$(2) \Rightarrow(1)$. For any $x \in U$, by (2), we have 
TABLE 2: Svnas.

\begin{tabular}{cccc}
\hline$R$ & $x_{1}$ & $x_{2}$ & $x_{3}$ \\
\hline$x_{1}$ & $(0,0,1)$ & $(0.3,0.1,0.6)$ & $(1,0,0.4)$ \\
$x_{2}$ & $(0,0.2,0.4)$ & $(0.6,0.5,1)$ & $(0.6,0,1)$ \\
$x_{3}$ & $(1,0,1)$ & $(1,0.5,1)$ & $(1,0,0)$ \\
\hline
\end{tabular}

TABle 3: The upper and lower approximation.

\begin{tabular}{lcc}
\hline & $\underline{R}(A)$ & $\bar{R}(A)$ \\
\hline$x_{1}$ & $(0.4,1,1)$ & $(0.3,0,0.6)$ \\
$x_{2}$ & $(1,1,0.6)$ & $(0.6,0.2,0.4)$ \\
$x_{3}$ & $(0,1,1)$ & $(1,0,1)$ \\
\hline
\end{tabular}

$$
\begin{aligned}
R_{T}(x, x) & =\bigvee_{y \notin U \dashv x\}} R_{T}(x, y) \\
& =\underline{R}(U-\{x\})_{F}(x) \geq\left(\top_{U \dashv x\}}\right)_{F}(x)=1, \\
1-R_{I}(x, x) & =\bigvee_{y \notin U \dashv x\}}\left(1-R_{I}(x, y)\right) \\
& =\underline{R}(U-\{x\})_{I}(x) \geq\left(\top_{U \dashv x\}}\right)_{I}(x)=1 \\
& \Rightarrow R_{I}(x, x)=0, \\
R_{F}(x, x) & =\bigwedge_{y \notin U\{x\}} R_{F}(x, y) \\
& =\underline{R}(U-\{x\})_{T}(x) \leq\left(\top_{U\{x\}}\right)_{T}(x)=0 .
\end{aligned}
$$

Hence, $R$ is reflexive.

$(2) \Leftrightarrow(3)$. It can be concluded from Theorem 1 (4).

The following theorem presents a characterization on the approximation operators generated by transitive Svnas.

Theorem 3. Let $(U, R)$ be an Svnas. Then the following three are equivalent:

(1) $R$ is transitive.

(2) For each $A \in P(U)$ and $x \in U$,

$$
\begin{aligned}
& \underline{R}(A)_{T}(x)=\bigvee_{B \subseteq A}\left(\underline{R}(B)_{T}(x) \bigwedge \bigwedge_{y \in B} \underline{R}(B)_{T}(y)\right), \\
& \underline{R}(A)_{I}(x)=\bigwedge_{B \subseteq A}\left(\underline{R}(B)_{I}(x) \text { scale } 190 \% \vee \bigvee_{y \in B} \underline{R}(B)_{I}(y)\right), \\
& \underline{R}(A)_{F}(x)=\bigwedge_{B \subseteq A}\left(\underline{R}(B)_{F}(x) \text { scale } 190 \% \vee \bigvee_{y \in B} \underline{R}(B)_{F}(y)\right) .
\end{aligned}
$$

(3) For each $A \in P(U)$ and $x \in U$,

$$
\begin{aligned}
& \bar{R}(A)_{T}(x)=\bigwedge_{A \subseteq B}\left(\bar{R}(B)_{T}(x) \text { scale } 190 \% \mathrm{~V} \bigvee_{y \notin B} \bar{R}(B)_{T}(y)\right), \\
& \bar{R}(A)_{I}(x)=\bigvee_{A \subseteq B}\left(\bar{R}(B)_{I}(x) \bigwedge \bigwedge_{y \notin B} \bar{R}(B)_{I}(y)\right), \\
& \bar{R}(A)_{F}(x)=\bigvee_{A \subseteq B}\left(\bar{R}(B)_{F}(x) \bigwedge \bigwedge_{y \notin B} \bar{R}(B)_{F}(y)\right) .
\end{aligned}
$$

Proof. $\quad(1) \Rightarrow(2)$. Let $A \in P(U)$ and $x \in U$.

(i) For any $B \subseteq A$, we have $\underline{R}(B)_{T}(x) \leq \underline{R}(A)_{T}(x)$ and so

$$
\begin{aligned}
\bigvee_{B \subseteq A}\left(\underline{R}(B)_{T}(x) \bigwedge \bigwedge_{y \in B} \underline{R}(B)_{T}(y)\right) & \leq \bigvee_{B \subseteq A}\left(\underline{R}(B)_{T}(x)\right) \\
& =\underline{R}(A)_{T}(x) .
\end{aligned}
$$

Conversely, let $\alpha=\underline{R}(A)_{T}(x)=\vee_{y \notin A} R_{F}(x, y)$; then $R_{F}(x, y) \geq \alpha$ for any $y \notin A$. Take $B_{x}=\{z \in U \mid$ $\left.R_{F}(x, z)<\alpha\right\}$; then $B_{x} \subseteq A$. It follows that

$$
\begin{array}{r}
\underline{R}\left(B_{x}\right)_{T}(x)=\bigwedge_{y \notin B_{x}} R_{F}(x, y) \geq \alpha, \\
\bigwedge_{y \in B_{x}} \underline{R}\left(B_{x}\right)_{T}(y)=\bigwedge_{y \in B_{x}} \bigwedge_{z \notin B_{x}} R_{F}(y, z) .
\end{array}
$$

Note that, for any $y \in B_{x}, z \notin B_{x}$, we have $R_{F}(x, y)<\alpha, R_{F}(x, z) \geq \alpha$. Since $R$ is transitive, we have $\quad R_{F}(x, y) \vee R_{F}(y, z) \geq R_{F}(x, z) \geq \alpha$, which means that $R_{F}(y, z) \geq \alpha$. So,

$$
\bigwedge_{y \in B_{x}} \underline{R}\left(B_{x}\right)_{T}(y)=\bigwedge_{y \in B_{x}} \bigwedge_{z \notin B_{x}} R_{F}(y, z) \geq \alpha,
$$

and then

$$
\begin{aligned}
& \bigvee_{B \subseteq A}\left(\underline{R}(B)_{T}(x) \bigwedge \bigwedge_{y \in B} \underline{R}(B)_{T}(y)\right) \\
& \geq \underline{R}\left(B_{x}\right)_{T}(x) \bigwedge \bigwedge_{y \in B_{x}} \underline{R}\left(B_{x}\right)_{T}(y) \geq \alpha=\underline{R}(A)_{T}(x) .
\end{aligned}
$$

Hence,

$$
\bigvee_{B \subseteq A}\left(\underline{R}(B)_{T}(x) \bigwedge \bigwedge_{y \in B} \underline{R}(B)_{T}(y)\right)=\underline{R}(A)_{T}(x) .
$$

(ii) For any $B \subseteq A$, we have $\underline{R}(B)_{I}(x) \geq \underline{R}(A)_{I}(x)$ and so

$$
\begin{aligned}
& \bigwedge_{B \subseteq A}\left(\underline{R}(B)_{I}(x) \text { scale } 190 \% \vee \bigvee_{y \in B} \underline{R}(B)_{I}(y)\right) \\
& \geq \bigwedge_{B \subseteq A}\left(\underline{R}(B)_{I}(x)\right)=\underline{R}(A)_{I}(x) .
\end{aligned}
$$

Conversely, let $\alpha=\underline{R}(A)_{I}(x)=\vee_{y \notin A}\left(1-R_{I} \quad(x\right.$, $y)$ ); then $1-R_{I}(x, y) \leq \alpha$ for any $y \notin A$. Take $B_{x}=$ $\left\{z \in U \mid 1-R_{I}(x, z)>\alpha\right\}$; then $B_{x} \subseteq A$. It follows that

$$
\begin{aligned}
\underline{R}\left(B_{x}\right)_{I}(x) & =\bigvee_{y \notin B_{x}}\left(1-R_{I}(x, y)\right) \leq \alpha, \\
\bigvee_{y \in B_{x}} \underline{R}\left(B_{x}\right)_{I}(y) & =\bigvee_{y \in B_{x}} \bigvee_{z \notin B_{x}}\left(1-R_{I}(y, z)\right) .
\end{aligned}
$$

Note that, for any $y \in B_{x}, z \notin B_{x}$, we have $1-R_{I}(x, y)>\alpha, 1-R_{I}(x, z) \leq \alpha$. Since $R$ is transitive, we have $\left(1-R_{I}(x, y)\right) \wedge$ $\left(1-R_{I}(y, z)\right) \leq\left(1-R_{I}(x, z)\right) \leq \alpha$, which means that $1-R_{I}(y, z) \leq \alpha$. So, 
$\bigvee_{y \in B_{x}} \underline{R}\left(B_{x}\right)_{I}(y)=\bigvee_{y \in B_{x}} \bigvee_{z \notin B_{x}}\left(1-R_{I}(y, z)\right) \leq \alpha$,

and then

$$
\begin{aligned}
& \bigwedge_{B \subseteq A}\left(\underline{R}(B)_{I}(x) \bigvee \bigvee_{y \in B} \underline{R}(B)_{I}(y)\right) \\
& \leq \underline{R}\left(B_{x}\right)_{I}(x) \bigvee \bigvee_{y \in B_{x}} \underline{R}\left(B_{x}\right)_{I}(y) \leq \alpha=\underline{R}(A)_{I}(x)
\end{aligned}
$$

Hence,

$$
\bigwedge_{B \subseteq A}\left(\underline{R}(B)_{I}(x) \bigvee \bigvee_{y \in B} \underline{R}(B)_{I}(y)\right)=\underline{R}(A)_{I}(x) .
$$

(iii) For any $B \subseteq A$, we have $\underline{R}(B)_{F}(x) \geq \underline{R}(A)_{F}(x)$ and so

$$
\begin{aligned}
\bigwedge_{B \subseteq A}\left(\underline{R}(B)_{F}(x) \bigvee \bigvee_{y \in B} \underline{R}(B)_{F}(y)\right) & \geq \bigwedge_{B \subseteq A}\left(\underline{R}(B)_{F}(x)\right) \\
& =\underline{R}(A)_{F}(x) .
\end{aligned}
$$

Conversely, let $\alpha=\underline{R}(A)_{F}(x)=\vee_{y \notin A} R_{T}(x, y)$; then $R_{T}(x, y) \leq \alpha$ for any $y \notin A$. Take $B_{x}=\left\{z \in U \mid R_{T}(x, z)>\alpha\right\}$; then $B_{x} \subseteq A$. It follows that

$$
\begin{aligned}
\underline{R}\left(B_{x}\right)_{F}(x) & =\bigvee_{y \notin B_{x}} R_{T}(x, y) \leq \alpha, \\
\bigvee_{y \in B_{x}} \underline{R}\left(B_{x}\right)_{F}(y) & =\bigvee_{y \in B_{x}} \bigvee_{z \notin B_{x}} R_{T}(y, z) .
\end{aligned}
$$

Note that, for any $y \in B_{x}, z \notin B_{x}$, we have $R_{T}(x, y)>\alpha, R_{T}(x, z) \leq \alpha$. Since $R$ is transitive, we have $R_{T}(x, y) \wedge R_{T}(y, z) \leq R_{T}(x, z) \leq \alpha$, which means that $R_{T}(y, z) \leq \alpha$. So,

$$
\bigvee_{y \in B_{x}} \underline{R}\left(B_{x}\right)_{F}(y)=\bigvee_{y \in B_{x}} \bigvee_{z \notin B_{x}} R_{T}(y, z) \leq \alpha,
$$

and then

$$
\begin{aligned}
\bigwedge_{B \subseteq A}\left(\underline{R}(B)_{F}(x) \bigvee \bigvee_{y \in B} \underline{R}(B)_{F}(y)\right) & \leq \underline{R}\left(B_{x}\right)_{F}(x) \bigvee \bigvee_{y \in B_{x}} \underline{R}\left(B_{x}\right)_{F}(y) \\
& \leq \alpha=\underline{R}(A)_{F}(x) .
\end{aligned}
$$

Hence,

$$
\bigwedge_{B \subseteq A}\left(\underline{R}(B)_{F}(x) \bigvee \bigvee_{y \in B} \underline{R}(B)_{F}(y)\right)=\underline{R}(A)_{F}(x) .
$$

(2) $\Rightarrow(1)$. Let $x, y, z \in U$.

(i) Note that

$$
\begin{aligned}
& R_{T}(x, z)=\underline{R}(U-\{z\})_{F}(x) \\
& \stackrel{(2)}{=} \bigwedge_{A \subseteq U\{z\}}\left(\underline{R}(A)_{F}(x) \bigvee \bigvee_{u \in A} \underline{R}(A)_{F}(u)\right), \\
& R_{T}(x, y)=\underline{R}(U-\{y\})_{F}(x) \\
& \stackrel{(2)}{=} \bigwedge_{B \subseteq U\{y\}}\left(\underline{R}(B)_{F}(x) \bigvee \bigvee_{v \in B} \underline{R}(B)_{F}(v)\right), \\
& R_{T}(y, z)=\underline{R}(U-\{z\})_{F}(y) \\
& \stackrel{(2)}{=} \bigwedge_{C \subseteq U\{z\}}\left(\underline{R}(C)_{F}(y) \bigvee \bigvee_{w \in C} \underline{R}(C)_{F}(w)\right)
\end{aligned}
$$

Take any $A \subseteq U-\{z\}$; then $y \in A$ or $y \notin A$.

Case 1: if $y \in A$, then

$$
\begin{aligned}
& \underline{R}(A)_{F}(x) \bigvee \bigvee_{u \in A} \underline{R}(A)_{F}(u) \\
& \geq \bigvee_{u \in A} \underline{R}(A)_{F}(u), \text { by } y \in A \\
& =\underline{R}(A)_{F}(y) \bigvee \bigvee_{u \in A} \underline{R}(A)_{F}(u), \text { by } A \subseteq U-\{z\} \\
& \geq \bigwedge_{C \subseteq U\{z\}}\left(\underline{R}(C)_{F}(y) \bigvee \bigvee_{w \in C} \underline{R}(C)_{F}(w)\right) \\
& \geq \bigwedge_{B \subseteq U\{y\}}\left(\underline{R}(B)_{F}(x) \bigvee \bigvee_{v \in B} \underline{R}(B)_{F}(v)\right) \\
& \bigwedge_{C \subseteq U\{z\}}\left(\underline{R}(C)_{F}(y) \bigvee \bigvee_{w \in C} \underline{R}(C)_{F}(w)\right) .
\end{aligned}
$$

Case 2: if $y \notin A$, then $A \subseteq U-\{y\}$ and so

$$
\begin{aligned}
& \underline{R}(A)_{F}(x) \bigvee \bigvee_{u \in A} \underline{R}(A)_{F}(u), \text { by } A \subseteq U-\{y\} \\
& \geq \bigwedge_{B \subseteq U\{y\}}\left(\underline{R}(B)_{F}(x) \bigvee \bigvee_{v \in B} \underline{R}(B)_{F}(v)\right) \\
& \geq \bigwedge_{B \subseteq U\{y\}}\left(\underline{R}(B)_{F}(x) \bigvee \bigvee_{v \in B} \underline{R}(B)_{F}(v)\right) \\
& \bigwedge \bigwedge_{C \subseteq U\{z\}}\left(\underline{R}(C)_{F}(y) \bigvee \bigvee_{w \in C} \underline{R}(C)_{F}(w)\right) .
\end{aligned}
$$

By a combination of Cases 1 and 2, we obtain

$$
\begin{aligned}
& \bigwedge_{A \subseteq U \dashv z\}}\left(\underline{R}(A)_{F}(x) \bigvee \bigvee_{u \in A} \underline{R}(A)_{F}(u)\right) \\
& \geq \bigwedge_{B \subseteq U\{y\}}\left(\underline{R}(B)_{F}(x) \bigvee \bigvee_{v \in B} \underline{R}(B)_{F}(v)\right) \\
& \bigwedge \bigwedge_{C \subseteq U\{z\}}\left(\underline{R}(C)_{F}(y) \bigvee \bigvee_{w \in C} \underline{R}(C)_{F}(w)\right)
\end{aligned}
$$

that is, $R_{T}(x, z) \geq R_{T}(x, y) \wedge R_{T}(y, z)$, as desired.

(ii) Note that 


$$
\begin{aligned}
& 1-R_{I}(x, z)=\underline{R}(U-\{z\})_{I}(x) \\
& \stackrel{(2)}{=} \bigwedge_{A \subseteq U\{z\}}\left(\underline{R}(A)_{I}(x) \text { scale } 190 \% \vee \bigvee_{u \in A} \underline{R}(A)_{I}(u)\right), \\
& 1-R_{I}(x, y)=\underline{R}(U-\{y\})_{I}(x) \\
& \stackrel{(2)}{=} \bigwedge_{B \subseteq U\{y\}}\left(\underline{R}(B)_{I}(x) \text { scale } 190 \% \vee \bigvee_{v \in B} \underline{R}(B)_{I}(v)\right), \\
& 1-R_{I}(y, z)=\underline{R}(U-\{z\})_{I}(y) \\
& \stackrel{(2)}{=} \bigwedge_{C \subseteq U\{z\}}\left(\underline{R}(C)_{I}(y) \text { scale } 190 \% \vee \bigvee_{w \in C} \underline{R}(C)_{I}(w)\right) .
\end{aligned}
$$

Similar to (i), we can prove that $1-R_{I}(x, z) \geq\left(1-R_{I}(x, y)\right) \wedge\left(1-R_{I}(y, z)\right)$; that is, $R_{I}(x, z) \leq R_{I}(x, y) \vee R_{I}(y, z)$, as desired.

(iii) Note that

$$
\begin{aligned}
& R_{F}(x, z)=\underline{R}(U-\{z\})_{T}(x) \\
& \stackrel{(2)}{=} \bigvee_{A \subseteq U\{z\}}\left(\underline{R}(A)_{T}(x) \wedge \bigwedge_{u \in A} \underline{R}(A)_{T}(u)\right), \\
& R_{F}(x, y)=\underline{R}(U-\{y\})_{T}(x) \\
& \stackrel{(2)}{=} \bigvee_{B \subseteq U\{y\}}\left(\underline{R}(B)_{T}(x) \wedge \bigwedge_{v \in B} \underline{R}(B)_{T}(v)\right), \\
& R_{F}(y, z)=\underline{R}(U-\{z\})_{T}(y) \\
& \stackrel{(2)}{=} \bigvee_{C \subseteq U\{z\}}\left(\underline{R}(C)_{T}(y) \wedge \bigwedge_{w \in C} \underline{R}(C)_{T}(w)\right) .
\end{aligned}
$$

Take any $A \subseteq U-\{z\}$; then $y \in A$ or $y \notin A$.

Case 1: if $y \in A$, then

$$
\begin{aligned}
& \underline{R}(A)_{T}(x) \wedge \bigwedge_{u \in A} \underline{R}(A)_{T}(u) \\
& \leq \bigwedge_{u \in A} \underline{R}(A)_{T}(u), \text { by } y \in A \\
& =\underline{R}(A)_{T}(y) \wedge \bigwedge_{u \in A} \underline{R}(A)_{T}(u), \text { by } A \subseteq U-\{z\} \\
& \leq \bigvee_{C \subseteq U\{z\}}\left(\underline{R}(C)_{T}(y) \wedge \bigwedge_{w \in C} \underline{R}(C)_{T}(w)\right) \\
& \leq \bigvee_{B \subseteq U\{y\}}\left(\underline{R}(B)_{T}(x) \wedge \bigwedge_{v \in B} \underline{R}(B)_{T}(v)\right) \\
& \text { scale } 190 \% \vee \bigvee_{C \subseteq U \dashv z\}}\left(\underline{R}(C)_{T}(y) \wedge \bigwedge_{w \in C} \underline{R}(C)_{T}(w)\right) .
\end{aligned}
$$

Case 2: if $y \notin A$, then $A \subseteq U-\{y\}$ and so

$$
\begin{aligned}
& \underline{R}(A)_{T}(x) \wedge \bigwedge_{u \in A} \underline{R}(A)_{T}(u), \text { by } A \subseteq U-\{y\} \\
& \leq \bigvee_{B \subseteq U\{y\}}\left(\underline{R}(B)_{T}(x) \wedge \bigwedge_{v \in B} \underline{R}(B)_{T}(v)\right) \\
& \leq \bigvee_{B \subseteq U\{y\}}\left(\underline{R}(B)_{T}(x) \wedge \bigwedge_{v \in B} \underline{R}(B)_{T}(v)\right) \\
& \text { scale } 190 \% \vee \bigvee_{C \subseteq U \smile\{z\}}\left(\underline{R}(C)_{T}(y) \wedge \bigwedge_{w \in C} \underline{R}(C)_{T}(w)\right) .
\end{aligned}
$$

By a combination of Cases 1 and 2, we obtain

$$
\begin{aligned}
& \bigvee_{A \subseteq U \dashv z\}}\left(\underline{R}(A)_{T}(x) \wedge \bigwedge_{u \in A} \underline{R}(A)_{T}(u)\right) \\
\leq & \bigvee_{B \subseteq U\{y\}}\left(\underline{R}(B)_{T}(x) \wedge \bigwedge_{v \in B} \underline{R}(B)_{T}(v)\right) \\
\text { scale } 190 \% \vee & \bigvee_{C \subseteq U\{z\}}\left(\underline{R}(C)_{T}(y) \wedge \bigwedge_{w \in C} \underline{R}(C)_{T}(w)\right),
\end{aligned}
$$

that is, $R_{F}(x, z) \leq R_{F}(x, y) \vee R_{F}(y, z)$, as desired.

From (i)-(iii), we know that $R$ is transitive.

(2) $\Leftrightarrow$ (3). It can be concluded from Theorem 1 (4).

\section{Ordinary Single-Valued Neutrosophic Topological Space Induced by Single-Valued Neutrosophic Approximation Space}

In this section, we shall consider the OSvnt induced by Svnas through the ordinary single-valued neutrosophic lower approximation operator.

At first, we fix a subclass of ordinary single-valued neutrosophic topological spaces.

Definition 7. An OSvnts $(U, \tau)$ is said to be quasidiscrete if it fulfills the following:

$$
\begin{aligned}
\text { (OSvnt2s)for any } A_{j} & \in P(U)(j \in J), \\
\tau_{T}\left(\cap_{j \in J} A_{j}\right) & \geq \wedge_{j \in J} \tau_{T}\left(A_{j}\right), \\
\tau_{I}\left(\cap_{j \in J} A_{j}\right) & \leq \vee_{j \in J} \tau_{I}\left(A_{j}\right), \\
\tau_{F}\left(\cap_{j \in J} A_{j}\right) & \leq \vee_{j \in J} \tau_{F}\left(A_{j}\right) .
\end{aligned}
$$

It is not difficult to see that quasidiscrete OSvnts is an extension of quasidiscrete topological space [10].

Theorem 4. Let $(U, R)$ be an Svnas. Then the Svns $\tau_{R}$ on $P(U)$ is defined as follows: for any $A \in P(U)$,

$$
\begin{aligned}
& \left(\tau_{R}\right)_{T}(A)=\bigwedge_{x \in A} \underline{R}(A)_{T}(x), \\
& \left(\tau_{R}\right)_{I}(A)=\bigvee_{x \in A} \underline{R}(A)_{I}(x), \\
& \left(\tau_{R}\right)_{F}(A)=\bigvee_{x \in A} \underline{R}(A)_{F}(x),
\end{aligned}
$$

is a quasidiscrete OSvnt on $U$.

Proof. OSvnt1: it follows that

$$
\begin{aligned}
& \left(\tau_{R}\right)_{T}(\varnothing)=\bigwedge_{x \in \varnothing} \underline{R}(\varnothing)_{T}(x)=1, \\
& \left(\tau_{R}\right)_{I}(\varnothing)=\bigvee_{x \in \varnothing} \underline{R}(\varnothing)_{I}(x)=0, \\
& \left(\tau_{R}\right)_{F}(\varnothing)=\bigvee_{x \in \varnothing} \underline{R}(\varnothing)_{F}(x)=0, \\
& \left(\tau_{R}\right)_{T}(U)=\bigwedge_{x \in U} \underline{R}(U)_{T}(x)=1, \\
& \left(\tau_{R}\right)_{I}(U)=\bigvee_{x \in U} \underline{R}(U)_{I}(x)=0, \\
& \left(\tau_{R}\right)_{F}(U)=\bigvee_{x \in U} \underline{R}(U)_{F}(x)=0 .
\end{aligned}
$$


OSvnt2s: let $A_{j} \in P(U)(j \in J)$. Then

$$
\begin{aligned}
\bigwedge_{j \in J}\left(\tau_{R}\right)_{T}\left(A_{j}\right) & =\bigwedge_{j \in J} \bigwedge_{x_{j} \in A_{j}} \underline{R}\left(A_{j}\right)_{T}\left(x_{j}\right) \\
& \leq \bigwedge_{j \in J} \bigwedge_{x \in \cap_{j \in J} A_{j}} \underline{R}\left(A_{j}\right)_{T}(x), \text { by Theorem 1(3) } \\
& =\bigwedge_{x \in \cap_{j \in J} A_{j}} \underline{R}\left(\cap_{j \in J} A_{j}\right)_{T}(x)=\left(\tau_{R}\right)_{T}\left(\cap_{j \in J} A_{j}\right), \\
\bigvee_{j \in J}\left(\tau_{R}\right)_{I}\left(A_{j}\right) & =\bigvee_{j \in J} \bigvee_{x_{j} \in A_{j}} \underline{R}\left(A_{j}\right)_{I}\left(x_{j}\right) \\
& \geq \bigvee_{j \in J} \bigvee_{x \in \cap_{j \in J} A_{j}} \underline{R}\left(A_{j}\right)_{I}(x), \text { by Theorem } 1(3) \\
& =\bigvee_{x \in \cap_{j \in J} A_{j}} \underline{R}\left(\cap_{j \in J} A_{j}\right)_{I}(x)=\left(\tau_{R}\right)_{I}\left(\cap_{j \in J} A_{j}\right) .
\end{aligned}
$$

Similarly, we can prove that $\vee_{j \in J}\left(\tau_{R}\right)_{F}\left(A_{j}\right) \geq$ $\left(\tau_{R}\right)_{F}\left(\cap_{j \in J} A_{j}\right)$.

OSvnt3: let $A_{j} \in P(U)(j \in J)$. Then it follows by Theorem 1 (2) that

$$
\begin{aligned}
& \bigwedge_{j \in J}\left(\tau_{R}\right)_{T}\left(A_{j}\right)=\bigwedge_{j \in J} \bigwedge_{x_{j} \in A_{j}} \underline{R}\left(A_{j}\right)_{T}\left(x_{j}\right), \\
& \leq \bigwedge_{j \in J} \bigwedge_{x_{j} \in A_{j}} \underline{R}\left(\cup_{j \in J} A_{j}\right)_{T}\left(x_{j}\right) \\
& =\bigwedge_{x \in \cup_{j \in J} A_{j}} \underline{R}\left(\cup_{j \in J} A_{j}\right)_{T}(x)=\left(\tau_{R}\right)_{T}\left(\cup_{j \in J} A_{j}\right), \\
& \bigvee_{j \in J}\left(\tau_{R}\right)_{I}\left(A_{j}\right)=\bigvee_{j \in J} \bigvee_{x_{j} \in A_{j}} \underline{R}\left(A_{j}\right)_{I}\left(x_{j}\right), \\
& \geq \bigvee_{j \in J} \bigvee_{x_{j} \in A_{j}} \underline{R}\left(\cup_{j \in J} A_{j}\right)_{I}\left(x_{j}\right) \\
& =\bigvee_{x \in \cup_{j \in J} A_{j}} \underline{R}\left(\cup_{j \in J} A_{j}\right)_{I}(x)=\left(\tau_{R}\right)_{I}\left(\cup_{j \in J} A_{j}\right) .
\end{aligned}
$$

Similarly, we can prove that $\vee_{j \in J}\left(\tau_{R}\right)_{F}\left(A_{j}\right)=\left(\tau_{R}\right)_{F}\left(\cup_{j \in J} A_{j}\right)$.

Remark 3. The definition of $\tau_{R}(A)$ is an interpretation of the fact that " $A$ is contained in its lower approximation."

\section{Single-Valued Neutrosophic Approximation Space Induced by Ordinary Single-Valued Neutrosophic Topological Space}

In this section, we shall consider the Svnas induced by OSvnt.

Theorem 5. Let $(U, \tau)$ be an OSvnts. Then the Svnr $R_{\tau}$ on $U$ is defined as follows: for any $(x, y) \in U \times U$,

$$
\begin{aligned}
& \left(R_{\tau}\right)_{T}(x, y)=\bigwedge_{(x, y) \in A \times A^{c}} \tau_{F}(A), \\
& \left(R_{\tau}\right)_{I}(x, y)=\bigvee_{(x, y) \in A \times A^{c}}\left(1-\tau_{I}(A)\right), \\
& \left(R_{\tau}\right)_{F}(x, y)=\bigvee_{(x, y) \in A \times A^{c}} \tau_{T}(A),
\end{aligned}
$$

is reflexive and transitive.
Proof. Reflexivity: it follows that

$$
\begin{aligned}
& \left(R_{\tau}\right)_{T}(x, x)=\bigwedge_{(x, x) \in A \times A^{c}} \tau_{F}(A)=1, \\
& \left(R_{\tau}\right)_{I}(x, x)=\bigvee_{(x, x) \in A \times A^{c}}\left(1-\tau_{I}(A)\right)=0, \\
& \left(R_{\tau}\right)_{F}(x, x)=\bigvee_{(x, x) \in A \times A^{c}} \tau_{T}(A)=0 .
\end{aligned}
$$

Transitivity: let $x, y, z \in U$.

(i) Note that

$$
\begin{aligned}
& \left(R_{\tau}\right)_{T}(x, y)=\bigwedge_{(x, y) \in A \times A^{c}} \tau_{F}(A), \\
& \left(R_{\tau}\right)_{T}(y, z)=\bigwedge_{(y, z) \in B \times B^{c}} \tau_{F}(B), \\
& \left(R_{\tau}\right)_{T}(x, z)=\bigwedge_{(x, z) \in D \times D^{c}} \tau_{F}(D) .
\end{aligned}
$$

Take any $D \in P(U)$ with $(x, z) \in D \times D^{c}$; then $y \in D$ or $y \in D^{c}$.

Case 1: if $y \in D$, then $(y, z) \in D \times D^{c}$. So,

$\left(R_{\tau}\right)_{T}(x, y) \wedge\left(R_{\tau}\right)_{T}(y, z)$

$\leq\left(R_{\tau}\right)_{T}(y, z)=\bigwedge_{(y, z) \in B \times B^{c}} \tau_{F}(B) \leq \tau_{F}(D)$.

Case 2: if $y \in D^{c}$, then $(x, y) \in D \times D^{c}$. So,

$\left(R_{\tau}\right)_{T}(x, y) \wedge\left(R_{\tau}\right)_{T}(y, z)$

$\leq\left(R_{\tau}\right)_{T}(x, y)=\bigwedge_{(x, y) \in A \times A^{c}} \tau_{F}(A) \leq \tau_{F}(D)$.

By a combination of Cases 1 and 2, we obtain that

$$
\begin{aligned}
& \left(R_{\tau}\right)_{T}(x, y) \wedge\left(R_{\tau}\right)_{T}(y, z) \\
& \leq \bigwedge_{(x, z) \in D \times D^{c}} \tau_{F}(D)=\left(R_{\tau}\right)_{T}(x, z) .
\end{aligned}
$$

(ii) Note that

$$
\begin{aligned}
& \left(R_{\tau}\right)_{I}(x, y)=\bigvee_{(x, y) \in A \times A^{c}}\left(1-\tau_{I}(A)\right), \\
& \left(R_{\tau}\right)_{I}(y, z)=\bigvee_{(y, z) \in B \times B^{c}}\left(1-\tau_{I}(B)\right), \\
& \left(R_{\tau}\right)_{I}(x, z)=\bigvee_{(x, z) \in D \times D^{c}}\left(1-\tau_{I}(D)\right) .
\end{aligned}
$$

Take any $D \in P(U)$ with $(x, z) \in D \times D^{c}$; then $y \in D$ or $y \in D^{c}$.

Case 1: if $y \in D$, then $(y, z) \in D \times D^{c}$. So,

$$
\begin{aligned}
& \left(R_{\tau}\right)_{I}(x, y) \vee\left(R_{\tau}\right)_{I}(y, z) \\
& \geq\left(R_{\tau}\right)_{I}(y, z)=\bigvee_{(y, z) \in B \times B^{c}}\left(1-\tau_{I}(B)\right) \\
& \geq\left(1-\tau_{I}(D)\right) .
\end{aligned}
$$

Case 2: if $y \in D^{c}$, then $(x, y) \in D \times D^{c}$. So,

$$
\begin{aligned}
& \left(R_{\tau}\right)_{I}(x, y) \vee\left(R_{\tau}\right)_{I}(y, z) \\
& \geq\left(R_{\tau}\right)_{I}(x, y)=\bigvee_{(x, y) \in A \times A^{c}}\left(1-\tau_{I}(A)\right) \\
& \geq\left(1-\tau_{I}(D)\right) .
\end{aligned}
$$


By a combination of Cases 1 and 2, we obtain that

$$
\begin{aligned}
\left(R_{\tau}\right)_{I}(x, y) & \vee\left(R_{\tau}\right)_{I}(y, z) \\
& \geq \bigvee_{(x, z) \in D \times D^{c}}\left(1-\tau_{I}(D)\right) \\
& =\left(R_{\tau}\right)_{I}(x, z) .
\end{aligned}
$$

(iii) Similar to (ii), one can prove that $\left(R_{\tau}\right)_{F}(x, y) \vee\left(R_{\tau}\right)_{F}(y, z) \geq\left(R_{\tau}\right)_{F}(x, z)$.

Remark 4. Note that neither of the topological conditions (OSvnt1)-(OSvnt3) is used in the above theorem. Hence, it can be extended to any single-valued neutrosophic relation on $P(U)$.

\section{One-to-One Correspondence between Reflexive and Transitive Single-Valued Neutrosophic Approximation Spaces and Quasidiscrete Ordinary Single-Valued Neutrosophic Topological Spaces}

In this section, we prove that there is a one-to-one correspondence between reflexive and transitive Svnas and quasidiscrete OSvnts.

Theorem 6. Let $(U, R)$ be an Svnas. Then $R_{\tau_{R}} \sqsupseteq R$, and $R_{\tau_{R}}=$ $R$ if $R$ is reflexive and transitive.

Proof. (1) For $x, y \in U$,

$$
\begin{aligned}
\left(R_{\tau_{R}}\right)_{T}(x, y) & =\bigwedge_{(x, y) \in A \times A^{c}}\left(\tau_{R}\right)_{F}(A) \\
& =\bigwedge_{(x, y) \in A \times A^{c}} \bigvee_{z \in A} \underline{R}(A)_{F}(z) \\
& =\bigwedge_{(x, y) \in A \times A^{c}} \bigvee_{z \in A} \bigvee_{w \notin A} R_{T}(z, w)
\end{aligned}
$$

$$
\left(R_{\tau_{R}}\right)_{T}(x, y) \leq R_{T}(x, y) \Leftrightarrow \forall \alpha \in[0,1), \quad \alpha<\left(R_{\tau_{R}}\right)_{T}(x, y) \text { implies } \alpha<R_{T}(x, y) .
$$

We assume that there is an $\alpha_{0} \in[0,1)$ such that $\alpha_{0}<\left(R_{\tau_{R}}\right)_{T}(x, y)$ but $\alpha_{0} \geq R_{T}(x, y)$. Putting $A_{0}=\left\{z \in U \mid R_{T}(x, z)>\alpha_{0}\right\}$, by reflexivity of $R$, we have $R_{T}(x, x)=1>\alpha_{0}$, so $x \in A_{0}$, and by $\alpha_{0} \geq R_{T}(x, y)$ we have $y \in\left(A_{0}\right)^{c}$. This means that $(x, y) \in A_{0} \times\left(A_{0}\right)^{c}$. From

$$
\alpha_{0}<\left(R_{\tau_{R}}\right)_{T}(x, y)=\bigwedge_{(x, y) \in A \times A^{c}} \bigvee_{(z, w) \in A \times A^{c}} R_{T}(z, w),
$$

$$
\begin{aligned}
& =\bigwedge_{(x, y) \in A \times A^{c}} \bigvee_{(z, w) \in A \times A^{c}} R_{T}(z, w) \text {, taking } z=x, w=y \\
& \geq \bigwedge_{(x, y) \in A \times A^{c}} R_{T}(x, y)=R_{T}(x, y), \\
& \left(R_{\tau_{R}}\right)_{I}(x, y)=\bigvee_{(x, y) \in A \times A^{c}}\left(1-\left(\tau_{R}\right)_{I}(A)\right) \\
& =\bigvee_{(x, y) \in A \times A^{c}}\left(1-\bigvee_{z \in A} \underline{R}(A)_{I}(z)\right) \\
& =\bigvee_{(x, y) \in A \times A^{c}}\left(1-\bigvee_{z \in A} \bigvee_{w \notin A}\left(1-R_{I}(z, w)\right)\right) \\
& =\bigvee_{(x, y) \in A \times A^{c}} \bigwedge_{(z, w) \in A \times A^{c}} R_{I}(z, w) \text {, taking } z=x, w=y \\
& \leq \bigvee_{(x, y) \in A \times A^{c}} R_{I}(x, y)=R_{I}(x, y), \\
& \left(R_{\tau_{R}}\right)_{F}(x, y)=\bigvee_{(x, y) \in A \times A^{c}}\left(\tau_{R}\right)_{T}(A) \\
& =\bigvee_{(x, y) \in A \times A^{c}} \bigwedge_{z \in A} \underline{R}(A)_{T}(z) \\
& =\bigvee_{(x, y) \in A \times A^{c}} \bigwedge_{z \in A} \bigwedge_{w \notin A} R_{F}(z, w) \\
& =\bigvee_{(x, y) \in A \times A^{c}} \bigwedge_{(z, w) \in A \times A^{c}} R_{F}(z, w) \text {, taking } z=x, w=y \\
& \leq \bigvee_{(x, y) \in A \times A^{c}} R_{F}(x, y)=R_{F}(x, y) \text {. }
\end{aligned}
$$

Hence, $R_{\tau_{R}} \sqsupseteq R$.

(2) Let $R$ be reflexive and transitive and $x, y \in U$.

(i) Note that we know that there exists $(z, w) \in A_{0} \times\left(A_{0}\right)^{c}$ such that $R_{T}(z, w)>\alpha_{0}$; that is, $R_{T}(x, z)>\alpha_{0}$ and $R_{T}(x, w) \leq \alpha_{0}$. It follows by the transitivity that

$\alpha_{0}<R_{T}(x, z) \wedge R_{T}(z, w) \leq R_{T}(x, w) \leq \alpha_{0}$,

a contradiction! Therefore, $\alpha<\left(R_{\tau_{R}}\right)_{T}(x, y)$ always implies that $\alpha<R_{T}(x, y)$. Hence, $\left(R_{\tau_{R}}\right)_{T}(x, y) \leq R_{T}(x, y)$.

(ii) Note that

$$
\left(R_{\tau_{R}}\right)_{I}(x, y) \geq R_{I}(x, y) \Leftrightarrow \forall \alpha \in(0,1], \quad \alpha \leq R_{I}(x, y) \text { implies } \alpha \leq\left(R_{\tau_{R}}\right)_{I}(x, y) .
$$


We assume that there is an $\alpha_{0} \in(0,1]$ such that $\alpha_{0} \leq R_{I}(x, y)$ but $\alpha_{0}>\left(R_{\tau_{R}}\right)_{I}(x, y)$. Putting $A_{0}=\left\{z \in U \mid R_{I}(x, z)<\alpha_{0}\right\}$, by reflexivity of $R$, we have $R_{I}(x, x)=0<\alpha_{0}$, so $x \in A_{0}$, and by $\alpha_{0} \leq R_{I}(x, y)$ we have $y \in\left(A_{0}\right)^{c}$. This means that $(x, y) \in A_{0} \times\left(A_{0}\right)^{c}$. From

$\alpha_{0}>\left(R_{\tau_{R}}\right)_{I}(x, y)=\bigvee_{(x, y) \in A \times A^{c}} \bigwedge_{(z, w) \in A \times A^{c}} R_{I}(z, w)$,

we know that there exists $(z, w) \in A_{0} \times\left(A_{0}\right)^{c}$ such that $R_{I}(z, w)<\alpha_{0}$; that is, $R_{I}(x, z)<\alpha_{0}$ and $R_{I}(x, w) \geq \alpha_{0}$. It follows by the transitivity that

$\alpha_{0}>R_{I}(x, z) \vee R_{I}(z, w) \geq R_{I}(x, w) \geq \alpha_{0}$,

a contradiction! Therefore, $\alpha \leq R_{I}(x, y)$ always implies that $\alpha \leq\left(R_{\tau_{R}}\right)_{I}(x, y)$. Hence, $\left(R_{\tau_{R}}\right)_{I}(x, y) \geq R_{I}(x, y)$.

(iii) Similar to (ii), we can prove that $\left(R_{\tau_{R}}\right)_{F}(x, y) \geq R_{F}(x, y)$.

(i)-(iii) show that $R \sqsupseteq R_{\tau_{R}}$, and so $R_{\tau_{R}}=R$ by (1).

(3) If $R_{\tau_{R}}=R$, then it follows by Theorems 4 and 5 that $R$ is reflexive and transitive.

Theorem 7. Let $(U, R)$ be an OSvnts. Then $\tau_{R_{\tau}} \sqsupseteq \tau$, and $\tau_{R_{\tau}}=$ $\tau$ if $\tau$ is quasidiscrete.

Proof
(1) Let $A \in P(U)$. Then

$$
\begin{aligned}
\left(\tau_{R_{\tau}}\right)_{T}(A) & =\bigwedge_{x \in A} R_{\tau}(A)_{T}(x) \\
& =\bigwedge_{x \in A} \bigwedge_{y \in A^{c}}\left(R_{\tau}\right)_{F}(x, y) \\
& =\bigwedge_{(x, y) \in A \times A^{c}} \bigvee_{(x, y) \in B \times B^{c}} \tau_{T}(B) \text {, taking } B=A \\
& \geq \bigwedge_{(x, y) \in A \times A^{c}} \tau_{T}(A)=\tau_{T}(A), \\
\left(\tau_{R_{\tau}}\right)_{I}(A) & =\bigvee_{x \in A} R_{\tau}(A)_{I}(x) \\
& =\bigvee_{x \in A} \bigvee_{y \in A^{c}}\left(1-\left(R_{\tau}\right)_{I}(x, y)\right) \\
& =\bigvee_{(x, y) \in A \times A^{c}}\left(1-\bigvee_{(x, y) \in B \times B^{c}}\left(1-\tau_{I}(B)\right)\right) \\
& =\bigvee_{(x, y) \in A \times A^{c}} \bigwedge_{(x, y) \in B \times B^{c}} \tau_{I}(B), \text { taking } B=A \\
& \leq \bigvee_{(x, y) \in A \times A^{c}} \tau_{I}(A)=\tau_{I}(A) .
\end{aligned}
$$

Similarly, we can prove that $\left(\tau_{R_{\tau}}\right)_{F}(A) \leq \tau_{F}(A)$.

(2) Let $A \in P(U)$.

(i) Note that

$$
\left(\tau_{R_{\tau}}\right)_{T}(A) \leq \tau_{T}(A) \Leftrightarrow \forall \alpha \in[0,1), \quad \alpha<\left(\tau_{R_{\tau}}\right)_{T}(A) \text { implies } \alpha \leq \tau_{T}(A) .
$$

We assume that

$\alpha<\left(\tau_{R_{\tau}}\right)_{T}(A)=\bigwedge_{(x, y) \in A \times A^{c}} \bigvee_{(x, y) \in B \times B^{c}} \tau_{T}(B)$

Then, for any $(x, y) \in A \times A^{c}$, there is $B_{x y} \in P(U)$ such that $(x, y) \in B_{x y} \times\left(B_{x y}\right)^{c}$ and $\alpha<\tau_{T}\left(B_{x y}\right)$. Putting $B_{y}=\cup_{x \in A} B_{x y}$, by (OSvnt3), we have

$\tau_{T}\left(B_{y}\right)=\tau_{T}\left(\cup_{x \in A} B_{x y}\right) \geq \bigwedge_{x \in A} \tau_{T}\left(B_{x y}\right) \geq \alpha$.

Note that $A=\cap_{y \in A^{c}} B_{y}$ (indeed, if $z \in A$, then, for any $y \in A^{c}, z \in B_{z y} \subseteq B_{y}$, and so $z \in \cap_{y \in A^{c}} B_{y}$; hence, $A \subseteq \cap_{y \in A^{c}} B_{y}$; if $z \notin A$, then, for any $x \in A$, we have $(x, z) \in A \times A^{c}$, and then $z \notin B_{x z}$ so $z \notin B_{z}$, which means that $z \notin \cap_{y \in A^{c}} B_{y}$; hence, $\left.\cap_{y \in A^{c}} B_{y} \subseteq A\right)$; then it follows by OSvnt2s that

$\tau_{T}(A)=\tau_{T}\left(\cap_{y \in A^{c}} B_{y}\right) \geq \bigwedge_{y \in A^{c}} \tau_{T}\left(B_{y}\right) \geq \alpha$.

Therefore, $\left(\tau_{R_{\tau}}\right)_{T}(A) \leq \tau_{T}(A)$.

(ii) Note that

$$
\left(\tau_{R_{\tau}}\right)_{I}(A) \geq \tau_{I}(A) \Leftrightarrow \forall \alpha \in(0,1], \quad \alpha>\left(\tau_{R_{\tau}}\right)_{I}(A) \text { implies } \alpha \geq \tau_{I}(A) .
$$

We assume that

$$
\left(\tau_{R_{\tau}}\right)_{I}(A)=\bigvee_{(x, y) \in A \times A^{c}} \bigwedge_{(x, y) \in B \times B^{c}} \tau_{I}(B)<\alpha .
$$

Then, for any $(x, y) \in A \times A^{c}$, there is $B_{x y} \in P(U)$ such that $(x, y) \in B_{x y} \times\left(B_{x y}\right)^{c}$ and $\alpha>\tau_{I}\left(B_{x y}\right)$. Putting $B_{y}=\cup_{x \in A} B_{x y}$, by OSvnt3, we have 
$\tau_{I}\left(B_{y}\right)=\tau_{I}\left(\cup_{x \in A} B_{x y}\right) \leq \bigvee_{x \in A} \tau_{I}\left(B_{x y}\right) \leq \alpha$.

Note that $A=\cap \cap_{y \in A^{c}} B_{y}$; then it follows by OSvnt2s that

$\tau_{I}(A)=\tau_{I}\left(\cap_{y \in A^{c}} B_{y}\right) \leq \bigvee_{y \in A^{c}} \tau_{I}\left(B_{y}\right) \leq \alpha$.

Therefore, $\left(\tau_{R_{\tau}}\right)_{I}(A) \geq \tau_{I}(A)$.

(iii) Similar to (ii), we can prove that $\left(\tau_{R_{\tau}}\right)_{F}(A) \geq \tau_{F}(A)$.

(i)-(iii) show that $\tau_{R_{\tau}} \sqsubseteq \tau$, and so $\tau_{R_{\tau}}=\tau$ by (1).

(3) If $\tau_{R_{\tau}}=\tau$, then it follows by Theorems 4 and 5 that $\tau$ is quasidiscrete.

From Theorems 6 and 7, we obtain the following corollary.

Corollary 1. There is a one-to-one correspondence between reflexive and transitive Svnas and quasidiscrete OSvnts with the same underlying set.

Remark 5. We can give a similar discussion on Svnas and ordinary single-valued neutrosophic cotopology in [47] via the ordinary single-valued neutrosophic upper approximation operator.

\section{Conclusions}

In this paper, we presented a new model of neutrosophic rough sets. The difference between this model and the existing models is that, in our model, the original sets are ordinary subsets of $U$ and their approximations are singlevalued neutrosophic sets; however, in the existing models, the original sets and their approximations are all (singlevalued) neutrosophic sets. We also discussed the basic properties of the proposed rough sets and gave their relationships with Kim's ordinary single-valued neutrosophic topology. Particularly, we proved by our lower approximation operator that there is a one-to-one correspondence between reflexive and transitive single-valued neutrosophic approximation spaces and quasidiscrete ordinary singlevalued neutrosophic topological spaces. In the future work, we shall present a more general single-valued neutrosophic topology such that it can be regarded as an extension of bifuzzy topology in [49]. We will also consider the corresponding single-valued neutrosophic rough sets related to the new single-valued neutrosophic topology. Furthermore, from Remark 1, we know that when restricting single-valued neutrosophic sets to Pythagorean fuzzy sets, we can define a model of Pythagorean fuzzy rough sets. It is well known that Pythagorean fuzzy sets and (fuzzy) rough sets have been applied in many fields, particularly in multiple attribute decision-making $[9,16,52-55]$. Therefore, in the future, we will also consider the potential application of Pythagorean fuzzy rough sets.

\section{Data Availability}

No data were used to support this study.

\section{Conflicts of Interest}

The author declares no conflicts of interest.

\section{Authors' Contributions}

Qiu Jin and Kai Hu contributed the central idea, and all authors contributed to the writing and revisions.

\section{Acknowledgments}

This work was supported by the National Natural Science Foundation of China (nos. 11801248 and 11501278) and Natural Science Foundation of Shandong Province (no. ZR2020MA042), and the KeYan Foundation of Liaocheng University (318012030).

\section{References}

[1] F. Smarandache, "Neutrosophic set-a generialization of the intuitionistics fuzzy sets," International Journal of Pure and Applied Mathematics, vol. 24, pp. 287-297, 2005.

[2] H. B. Wang, F. Smarandache, Y. Zhang, and R. Sunderraman, "Single valued neutrosophic sets," Multispace Multistructure, vol. 4, pp. 410-413, 2010.

[3] L. A. Zadeh, "Fuzzy sets," Inform and Control, vol. 8, no. 1, pp. 338-353, 1965.

[4] K. T. Atanassov, "Intuitionistic fuzzy sets," Fuzzy Sets and Systems, vol. 20, no. 1, pp. 87-96, 1986.

[5] M. Abdel-Basset, M. Mohamed, A.-N. Hussien, and A. K. Sangaiah, "A novel group decision-making model based on triangular neutrosophic numbers," Soft Computing, vol. 22, no. 20, pp. 6629-6643, 2018.

[6] J. Ye, "A multicriteria decision-making method using aggregation operators for simplified neutrosophic sets," Journal of Intelligent \& Fuzzy Systems, vol. 26, no. 5, pp. 2459-2466, 2014.

[7] J. Ye, "A netting method for clustering-simplified neutrosophic information," Soft Computing, vol. 21, no. 24, pp. 7571-7577, 2017.

[8] P. Majudar and S. K. Samant, "On similarity and entropy of neutrosophic sets," Journal of Intelligent \& Fuzzy Systems, vol. 26, pp. 1245-1252, 2014.

[9] J. Li, H. L. Yang, and S. G. Li, "Three-way decision based on decision theoretic rough sets with single-valued neutrosophic information," International Journal of Machine Learning and Cybernetics, vol. 11, pp. 657-665, 2020.

[10] H.-L. Yang, C.-L. Zhang, Z.-L. Guo, Y.-L. Liu, and X. Liao, “A hybrid model of single valued neutrosophic sets and rough sets: single valued neutrosophic rough set model," Soft Computing, vol. 21, no. 21, pp. 6253-6267, 2017.

[11] S. Shao and X. Zhang, "Measures of probabilistic neutrosophic hesitant fuzzy sets and the application in reducing unnecessary evaluation processes," Mathematics, vol. 7, no. 7, p. $649,2019$.

[12] J. Q. Wang and X. H. Zhang, "Multigranulation single valued neutrosophic covering-based rough sets and their applications to multi-criteria group decision making, Iran," Journal of Fuzzy Systems, vol. 17, no. 5, pp. 109-126, 2020.

[13] X. Zhang, C. Bo, F. Smarandache, and J. Dai, "New inclusion relation of neutrosophic sets with applications and related lattice structure," International Journal of Machine Learning and Cybernetics, vol. 9, no. 10, pp. 1753-1763, 2018. 
[14] E. K. Zavadskas, R. Bausys, and I. Lescauskiene, "Multimoora under interval-valued neutrosophic sets as the basis for the quantitative heuristic evaluation methodology Hebin," Mathematics, vol. 1, no. 9, p. 66, 2021.

[15] D. S. Xu, X. L. Wei, and H. Ding, "A new method based on PROMETHEE and TODIM for multi-attribute decisionmaking with single-valued neutrosophic sets," Mathematics, vol. 10, no. 8, p. 1816, 2020.

[16] X. Peng and J. Dai, "A bibliometric analysis of neutrosophic set: two decades review from 1998 to 2017," Artificial Intelligence Review, vol. 53, no. 1, pp. 199-255, 2020.

[17] Z. A. Pawlak, "Rough sets," International Journal of Computer \& Information Sciences, vol. 11, no. 5, pp. 341-356, 1982.

[18] C. Zhang, D. Li, X. Kang, D. Song, A. K. Sangaiah, and S. Broumi, "Neutrosophic fusion of rough set theory: an overview," Computers in Industry, vol. 115, p. 103117, 2020.

[19] S. Broumi, F. Smarandache, and M. Dhar, "Rough neutrosophic sets," Italian Journal of Pure and Applied Mathematics, vol. 32, pp. 493-502, 2014.

[20] C. A. C. Sweety and I. Arockiarani, "Rough sets in neutrosophic approximation space," Annals of Fuzzy Mathematics and Informatics, vol. 13, no. 4, pp. 449-463, 2017.

[21] B. Y. Ling, H. L. Yang, and S. G. Li, "On characterization of (I, $N)$-single valued neutrosophic rough approximation operators," Soft Computing, vol. 23, no. 15, pp. 6065-6084, 2019.

[22] Y. Ma, W. Zhou, and Q. Wang, "Covering-based rough single valued neutrosophicsets," Neutrosophic Sets and Systems, vol. 17, pp. 3-9, 2017.

[23] H. Zhao and H.-Y. Zhang, "A result on single valued neutrosophic refined rough approximation operators1," Journal of Intelligent \& Fuzzy Systems, vol. 35, no. 3, pp. 3139-3146, 2018.

[24] H. Zhao and H.-Y. Zhang, "On hesitant neutrosophic rough set over two universes and its application," Artificial Intelligence Review, vol. 53, no. 6, pp. 4387-4406, 2020.

[25] A. Allam, M. Bakeir, and E. Tabl, "Some methods for generating topologies by relations," Bulletin of the Malaysian Mathematical Sciences Society, vol. 31, no. 1, pp. 35-45, 2018.

[26] J. Fang and P. Chen, "One-to-one correspondence between fuzzifying topologies and fuzzy preorders," Fuzzy Sets and Systems, vol. 158, no. 16, pp. 1814-1822, 2007.

[27] Q. Jin, L. Q. Li, and G. M. Lang, "p-regularity and p-regular modification in T-convergence spaces," Mathematics, vol. 4, no. 7, p. 370, 2019.

[28] Q. Jin, L. Li, Z. Ma, and B. Yao, "A note on the relationships between generalized rough sets and topologies," International Journal of Approximate Reasoning, vol. 130, pp. 292-296, 2021.

[29] L. Q. Li, Q. Jin, K. Hu, and F. F. Zhao, "The axiomatic characterizations onL-fuzzy covering-based approximation operators," International Journal of General Systems, vol. 46, no. 4, pp. 332-353, 2017.

[30] L. Li, Q. Jin, B. Yao, and J. Wu, "A rough set model based on fuzzifying neighborhood systems," Soft Computing, vol. 24, no. 8, pp. 6085-6099, 2020.

[31] L. Li, B. Yao, J. Zhan, and Q. Jin, " $L$-fuzzifying approximation operators derived from general L-fuzzifying neighborhood systems," International Journal of Machine Learning and Cybernetics, vol. 12, no. 5, pp. 1343-1367, 2021.

[32] W. Li, X. Xue, W. Xu, T. Zhan, and B. Fan, "Double-quantitative variable consistency dominance-based rough set approach," International Journal of Approximate Reasoning, vol. 124, pp. 1-26, 2020.
[33] B. Pang, J.-S. Mi, and Z.-Y. Xiu, " $L$-fuzzifying approximation operators in fuzzy rough sets," Information Sciences, vol. 480, pp. 14-33, 2019.

[34] Z. Pei, D. Pei, and L. Zheng, "Topology vs generalized rough sets," International Journal of Approximate Reasoning, vol. 52, no. 2, pp. 231-239, 2011.

[35] S. B. Sun, L. Q. Li, and K. Hu, "A new approach to rough set based on remote neighborhood systems," Mathematical Problems in Engineering, vol. 2019, Article ID 8712010, , 2019.

[36] S. B. Sun, L. Q. Li, K. Hu, and A. A. Ramadan, " $L$-fuzzy upper approximation operators associated with $L$-generalized fuzzy remote neighborhood systems of $L$-fuzzy points," AIMS Mathematics, vol. 5, no. 6, pp. 5638-5652, 2021.

[37] S. P. Tiwaria and K. S. Arun, "Fuzzy rough sets, fuzzy preorders and fuzzy topologies," Fuzzy Sets and Systems, vol. 210, pp. 63-68, 2013.

[38] H. Wu and G. Liu, "The relationships between topologies and generalized rough sets," International Journal of Approximate Reasoning, vol. 119, pp. 313-324, 2020.

[39] J. Zhan, H. Jiang, and Y. Yao, "Covering-based variable precision fuzzy rough sets with PROMETHEE-EDAS methods," Information Sciences, vol. 538, pp. 314-336, 2020.

[40] F. Zhao, Q. Jin, and L. Li, "The axiomatic characterizations on L-generalized fuzzy neighborhood system-based approximation operators," International Journal of General Systems, vol. 47, no. 2, pp. 155-173, 2018.

[41] F. Zhao and L. Li, "Axiomatization on generalized neighborhood system-based rough sets," Soft Computing, vol. 22, no. 18, pp. 6099-6110, 2018.

[42] F. F. Zhao, L. Q. Li, S. B. Sun, and Q. Jin, "Rough approximation operators based on quantale-valued fuzzy generalized neighborhood systems," Iran Journal of Fuzzy Systems, vol. 16, no. 6, pp. 53-63, 2019.

[43] W. Al-Omeri and S. Jafari, "On generalized closed cets and generalized pre-closed sets in neutrosophic topological spaces," Mathematics, vol. 1, no. 7, p. 1, 2019.

[44] F. G. Lupiáñez, "On neutrosophic sets and topology," Procedia Computer Science, vol. 120, pp. 975-982, 2017.

[45] Y.-L. Liu and H.-L. Yang, "Further research of single valued neutrosophic rough sets," Journal of Intelligent \& Fuzzy Systems, vol. 33, no. 3, pp. 1467-1478, 2017.

[46] R. Lowen, "Fuzzy topological spaces and fuzzy compactness," Journal of Mathematical Analysis and Applications, vol. 56, no. 3, pp. 621-633, 1976.

[47] J. H. Kim, F. Smarandache, J. G. Lee, and K. Hur, "Ordinary single valued neutrosophic topological spaces," Symmetry, vol. 11, no. 9, p. 1075, 2019.

[48] A. Sostak, "On a fuzzy topological structure," Supplemento ai Rendiconti del Circolo Matematico di Palermo, vol. 2, pp. 89-103, 1985.

[49] M. Ying, "A new approach for fuzzy topology (I)," Fuzzy Sets and Systems, vol. 39, no. 3, pp. 303-321, 1991.

[50] R. R. Yager, "Pythagorean membership grades in multicriteria decision making," IEEE Transactions on Fuzzy Systems, vol. 22, no. 4, pp. 958-965, 2014.

[51] Y. Y. Yao, "Combination of rough and fuzzy sets based on $\alpha$-level sets," Rough Sets and Data Mining, vol. 104, pp. 301-321, 1997.

[52] S. Shao and X. Zhang, "Multiobjective programming approaches to obtain the priority vectors under uncertain probabilistic dual hesitant fuzzy preference environment," International Journal of Computational Intelligence Systems, vol. 14, no. 1, pp. 1189-1207, 2021. 
[53] L. Wang, H. Garg, and N. Li, "Pythagorean fuzzy interactive Hamacher power aggregation operators for assessment of express service quality with entropy weight," Soft Computing, vol. 25, no. 2, pp. 973-993, 2021.

[54] L. Wang and H. Garg, "Algorithm for multiple attribute decision-making with interactive archimedean norm operations under pythagorean fuzzy uncertainty," International Journal of Computational Intelligence Systems, vol. 14, no. 1, pp. 503-527, 2021.

[55] X. Zhang and Z. Xu, "Extension of TOPSIS to multiple criteria decision making with pythagorean fuzzy sets," International Journal of Intelligent Systems, vol. 29, no. 12, pp. 1061-1078, 2014. 